\title{
A spectroscopic analysis of the Trapezium Cluster stars
}

\author{
Sergio Simón-Díaz ${ }^{1}$, Artemio Herrero ${ }^{1,2}$, and César Esteban ${ }^{1,2}$ \\ ${ }^{1}$ Instituto de Astrofísica de Canarias, \\ C/Vía Láctea s/n, E-38205 La Laguna, La Laguna, España \\ ${ }^{2}$ Departamento de Astrofísica, Universidad de La Laguna, \\ Avda. Astrofísico Francisco Sánchez, s/n, E-38071 La Laguna, España
}

\begin{abstract}
We consider the Orion Nebula (M 42) within a project aimed at studying the interaction between massive stars and their surrounding ISM. This is an $\mathrm{H}$ II region ionized by $\theta^{1}$ Ori C, one of the four massive stars in the Trapezium Cluster. $\theta^{1}$ Ori $\mathrm{C}$ has the earliest spectral type (O7Vp) among them, emitting an ionizing flux several orders of magnitude larger than those of the other stars. We present a spectral analysis of the Trapezium Cluster stars to determine their stellar parameters. We use spectra between $4250-4750 \AA$ and compare them with synthetic spectra obtained by means of an updated version of FASTWIND that includes an approximated treatment of metal-line blanketing.
\end{abstract}

\section{Introduction}

H II regions offer the possibility to derive accurate chemical abundances at large distances, thanks to the radiative energies emerging from the nearby ionizing stars. These are young, massive stars, recently born from the ISM, and therefore should share the same chemical composition. However, abundance determinations have not always given the same results for stars and the ISM. Recent agreement in our Galaxy (Smartt \& Rolleston 1997) has been followed by consistency in M 33 (Monteverde, Herrero \& Lennon 2000) and differences in M 31 (Smartt et al. 2001). See, however, Daflon \& Cunha (these Proceedings).

Recent developments in stellar atmosphere models predict lower $T_{\text {eff }}$ and luminosities than previously accepted (see Herrero, these Proceedings). We plan to use FASTWIND (Santolaya-Rey, Puls \& Herrero 1997), to derive more accurate abundances and emergent fluxes from stars ionizing $\mathrm{H}$ II regions. The ionizing fluxes will be used as input to a program of radiative transfer in ionized media, to derive $\mathrm{H}$ II region abundances and compare with those of the ionizing stars.

\section{The Orion Nebula and the Trapezium Cluster}

The Orion Nebula is one of the best studied $\mathrm{H}$ II regions. Its proximity (450 pc) has allowed us to resolve its structure and physical properties, and to obtain its element abundances accurately (Esteban et al. 1998; O'Dell 2001). The Trapezium Cluster, located in the central part of the nebula, is formed by four 
massive stars of OB spectral type. The star $\theta^{1}$ Ori C is the hottest one (O7Vp, Conti 1972), producing an ionizing flux several orders of magnitude greater than the other three $\left(\theta^{1}\right.$ Ori $\left.A, B, D\right)$. It is the star ionizing the nebula.

\section{Spectral characteristics of the Trapezium Cluster stars}

\section{1. $\theta^{1}$ Ori B}

Due to fast rotation, $\mathrm{H}$ and $\mathrm{He}$ lines are very broad and metal lines are diluted. $\mathrm{H}$ and $\mathrm{He} \mathrm{I}$ lines are strong and no $\mathrm{He}$ II lines appear in the spectrum. The star seems to be a B5V star with a high projected rotational velocity (200$250 \mathrm{~km} \mathrm{~s}^{-1}$ ).

\section{2. $\boldsymbol{\theta}^{1}$ Ori A, D}

These $\mathrm{B} 0.5 \mathrm{~V}$ stars show $\mathrm{Si}, \mathrm{Mg}, \mathrm{O}, \mathrm{C}$ and $\mathrm{N}$ lines, being perfect targets for the stellar abundance study proposed.

\section{3. $\theta^{1}$ Ori C}

We have found those spectral features mentioned in the literature for this star, but also evidence suggesting a composite spectrum (broad He I, already known, together with possible Si III lines). More work is needed to understand the nature of this star, before we can apply spherically symmetric mass-losing models to derive its stellar parameters.

\section{Data analysis}

For the analysis we use FASTWIND, a stellar atmosphere code developed by Santolaya-Rey et al. (1997), that has been updated by Puls et al. (2002), considering metal-line effect over the atmosphere structure and emergent flux (lineblanketing and -blocking). The parameters derived for $\theta^{1}$ Ori A are $T_{\text {eff }}=30000$ $\pm 2500 \mathrm{~K}$ and $\log g=4.2 \pm 0.2$, and for $\theta^{1}$ Ori D, $T_{\text {eff }}=32500 \mathrm{~K}$ and $\log g=4.4$ (same errors). Other analyses are presently under work.

\section{References}

Conti, P.S. 1972, ApJ (Letters) 174, L79

Esteban, C., Peimbert, M., Torres-Peimbert, S., et al. 1998, MNRAS 295, 401

Monteverde, M.I., Herrero, A., Lennon, D.J. 2000, ApJ 545, 813

O'Dell, C.R. 2001, Ann. Review Astron. Astrophys. 39, 99

Puls, J. et al. 2002, in preparation

Santolaya-Rey, E., Puls, J., Herrero, A. 1997, A\&A 323, 488

Smartt, S.J., Crowther, P.A., Dufton, P.L., et al. 2001, MNRAS 325, 257

Smartt, S.J., Rolleston, W.R.J. 1997, ApJ 481, 47 